\title{
Index of Sources
}

\section{Jewish Sources}

\author{
Biblical \\ Genesis \\ $1 \quad 184,189$ \\ 1:1 211n219 \\ 1:27 209 \\ 2:9 143 \\ 2:17 207n207 \\ $3126 n 87$ \\ 6210 \\ 11:1-9 171n42 \\ 14:14 105n281 \\ 37:2 155n190 \\ 49:14 XIII, 148n155
}

\section{Exodus}

3:2 211n219

3:8 247n364

3:14 251

3:15 144n143

13:16 283n116

19:5 282n108

20:1 211n219

20:20 211n219

23:21 287n145

24:12 188n115

28:17-22 74n145

31:18 191n124, 193n133

32:16 188n114

$34: 6 \quad 341$

Leviticus

23:17 339n152

Numbers

15:22 155n190

21:30 347n178

32:34 347n178

\section{Deuteronomy}

4:37 201n173

14:1 211

14:2 201n172

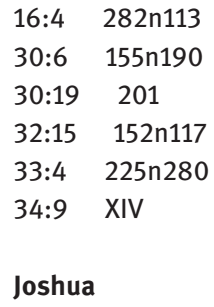

Joshua

13:9 347n178

$17 \quad 347 n 178$

\section{Samuel}

7:23 281n103

\section{Kings}

10:22 118n63

\section{Chronicles}

16:13 183n91

$17: 21 \quad 282$ n112

Nehemiah

$9: 2 \quad 183 n 91$

\section{Psalms}

$\begin{array}{lc}2: 4 & 137 n 125 \\ 16 & 290 n 161 \\ 16: 8 & 270 n 49 \\ 22: 23 & 183 n 91 \\ 45: 2 & 197 n 149 \\ 50: 1 & 339 n 148,340 n 152 \\ 68: 5 & 315 n 59 \\ 86 & 362 \\ 147 & 214 n 232\end{array}$

Proverbs

$1 \quad 185 \mathrm{n} 100$

9:17 116n48

Isaiah

9:1 109n13

Ә OpenAccess. (c) 2020, Moshe Idel, published by De Gruyter. (c) BY-NC-ND This work is licensed under the Creative Commons Attribution-NonCommercial-NoDerivatives 4.0 License. https://doi.org/10.1515/9783110599978-009 


$\begin{array}{ll}\text { 11:1-2 } & \text { XIV } \\ 11: 9 & 166 n 26 \\ 25: 8 & 148 \mathrm{n} 156 \\ 26: 2 & 183 n 91 \\ 28: 5 & 183 n 91 \\ 38: 16 & 284 n 127 \\ 40: 22 & 137 n 124 \\ 45: 19 & 183 n 91 \\ 52: 13 & 251 n 391 \\ 60: 21 & 183 n 91 \\ 61: 9 & 183 n 91 \\ 64: 9 & 183 n 91 \\ 65: 9 & 183 n 91\end{array}$

\section{Ezekiel}

$1312 \mathrm{n} 43$

1:26 232n309

$9: 4 \quad 313 n 49$

Daniel

\section{1:7 74n146 \\ 12:4 165n24 \\ 12:9 79n169}

Micah

$2: 13 \quad 284$

Zefania

3:9 251n389

\section{Zachariah}

1:7 74n150

9:10 216n239

\section{Ecclesiastes}

9:8 288n151

\section{Mishna}

Avot

- 4:5 258n10

Hagigah

- 2:1 111n24

\section{Babylonian Talmud}

Berakhot

- 6 a 280, 282n110

\begin{tabular}{|c|c|}
\hline$-7 a \quad 2$ & 284n119 \\
\hline$-28 b$ & $203 n 183$ \\
\hline$-29 a$ & $56 n 54$ \\
\hline$-58 b$ & $118 n 63$ \\
\hline \multicolumn{2}{|c|}{ Šabbat } \\
\hline$-146 a$ & $\mathrm{XV}, 126 \mathrm{n} 87$ \\
\hline \multicolumn{2}{|c|}{ Pesaḥim } \\
\hline$-54 a$ & $188 \mathrm{n} 113$ \\
\hline \multicolumn{2}{|c|}{ Hagigah } \\
\hline$-12 b$ & 138n127 \\
\hline$-13 a$ & $123 n 81$ \\
\hline \multicolumn{2}{|c|}{ Yebamot } \\
\hline$-63 b$ & $335 n 135$ \\
\hline$-103 b$ & 126n87 \\
\hline \multicolumn{2}{|c|}{ Baba'Meși'a' } \\
\hline$-31 b$ & 281n104 \\
\hline \multicolumn{2}{|c|}{ Babba' Batra' } \\
\hline$-16 b$ & $266 n 35$ \\
\hline 'Avodah & Zarah \\
\hline$-22 b$ & $126 n 87$ \\
\hline
\end{tabular}

\section{Midrash}

Genesis Rabbah

-10:6 XIV, 69n106

Mekhilta' de-Rashby on Exodus, eds. Yaakov N. Epstein and Ezra Z. Melamed (Jerusalem: Mekize Nirdamim, 1959)

-129-31 86n197

Midrash Šoḥer Tov 339n148

Midrash of Song of Songs

- 1:1, 159n4

Midrash Tanḥuma'

- pericope 'eqev, par. 7, 351n201

Sifri, Devarim

- 25, 281n105

Yalquț Šim 'oni

- 28; 31, 126n87

$-130,126 n 87$

$-247 ; 28,126 n 87$

\section{Hekhalot}

Hekhalot Rabbati

- 37 and 39, 351n201

Sefer Yeșirah, XIII, 46, 55n50, 56, 66-68, 70n113, 79, 86, 89, 93, 103-6, 109, 122n78, 142, 154, 161-62, 178-79, 181-82, 184, 189, 194n139, 195, 289, 321, 334, 336n136, 341

$-1: 2-8,196 n 148$ 
$-2: 6,188 n 111$

$-2: 8,86 n 196,247 n 367$

$-4: 3,153 n 178$

\section{Kabbalah}

Zohar, 80n170, 141, 150n168, 223, 268n44, 269n45, 272-75, 278, 279n93, 280, 283, 307, 327, 338, 341, 350, 354n214

- 3, 73a, 123n81

- 3, 175b, 283n117

$-3,130 \mathrm{~b}, 166 \mathrm{n} 25$

- 3, 262b-263a, 283n115

\section{Medieval}

\section{Abraham Abulafia}

Commentary on Iš Adam (in Mașref ha-Śekhel) - 49, 168n33, 336n137

Commentary on Sefer ha-'Edut, in: Mașref haŚekhel. ed. Gross (Jerusalem: 2001), 46, 328, 330, 332, 349

- 57, 46n4, 76n155, 84n188, 108n6, 344n169, 345n170, 346, 349n186, 352n205

- 58, 239n334, 349n187

$-60,229 n 298,332 n 121$

$-61,333 n 125,334,349 n 188$

$-75,352$ n206

$-76,168 n 35$

Commentary on Sefer ha-Hayyim, in: Mașref ha-Śekhel, ed. Gross (Jerusalem: 2001)

- 79, 348n185, 352n206

$-82,352 \mathrm{n} 206$

$-83,352$

Commentary on Sefer ha-Meliș (in Mașref haŚekhel), 110, 228n289, 264n29, 267n37

$-8,209 \mathrm{n} 211$

$-36,111 \mathrm{n} 20$

- 40, 77n157

- Ms. Roma, Angelica 38, fol. 9ạ, 119n66, 209n211, 214n229

Commentary on Sefer ha-Yašar, in: Mașref haŚekhel, ed. Gross (Jerusalem: 2001), $251 n 390$

-97-98, 347n180

- 98, 347n179

-99, 222n268, 346n173, 349n188

$-106,222$ n267
Commentary on Sefer Yeșirah (Jerusalem: Mossad ha-Rav Kook, 1984), 53n29, 121, 361

$-1-2,211 n 219$

$-3,122 n 78$

$-4,122 n 78$

$-8-9,204 n 192$

$-10,85 n 191$

$-18-20,103 n 268$

$-19,219 n 253,361$

$-20,230$ n 300

$-21,187 \mathrm{n} 106$

$-24,152 \mathrm{n} 176,232 \mathrm{n} 310$

$-31,315 n 63$

- 34-35, 316n64

Geț ha-Šemot, 142, 144, 147, 151, 192

$-1,206 n 203$

$-2,142 n 137$

$-3,142 \mathrm{n} 137$

$-4,187 n 106$

$-5-6,143 n 138$

$-7,59 n 70$

$-13,187 \mathrm{n} 106$

$-20,179 n 76$

$-21,314 n 51$

$-24,46 n 2$

$-30,142 \mathrm{n} 137$

$-31,142 \mathrm{n} 137$

$-32,142 \mathrm{n} 137$

$-33,69 n 109$

$-34,142 \mathrm{n} 137,187 \mathrm{n} 106$

$-35,142$ n137

$-38,179,179 n 79$

- 40, 142n137, 143n141, 206n203, 252n394

- 40-41, 101n257

- Ms. Oxford, Bodleian 1682, 101b, 193n134

Ḥayyei ha-Nefeš, 50n17, 89n208, 94, 132, 136n117, 171, 196n146, 216, 234n318, 285n134, 337, 359-60

$-3,135 n 117$

$-4,219$ n 256

$-5,265 n 32$

$-8,132 \mathrm{n} 110$

- 9, 116n47, 175n63, 222n268

$-10,116 n 47,209 n 214$

- 11, 200n169

$-12,114 n 36$

$-13,112 \mathrm{n} 30$

$-15,117 \mathrm{n} 57,222 \mathrm{n} 268$

$-16,211 \mathrm{n} 217$

$-18,57 n 61$ 
- 20, 187n106, 267n39, 360n7, 364n26

$-32,201 \mathrm{n} 170$

$-48,63 n 84$

$-59,333 n 125$

$-60,227 \mathrm{n} 287$

$-66,103$

$-72,360 n 7$

$-72-73,359 n 3$

$-73,359 n 4$

$-77,189 n 118,217 n 243$

$-79,189$ n118

$-80,171 n 42$

$-81-82,60 n 74$

- 83, 136n117, 204n192

$-87,313 n 46$

$-88,333 n 126$

$-89,150 \mathrm{n} 168$

$-90,150 \mathrm{n} 168$

$-92,127 n 93$

- 94-95, 281n106

$-97,333 n 126$

$-99,211 \mathrm{n} 219$

$-101,103$, n264

$-103,59 n 72$

$-114,243 n 355$

$-121,119 n 66$

$-121-22,319 n 75$

- 126-27, 217n243

$-128,233 n 310,252 n 394$

$-133,211 n 219$

- 134, 285n134, 336n136

$-142,228$ n 290

$-142-43,137 n 123$

$-143,59 n 71$

- 144-45, 147n154

$-147,178 n 74$

- 151, 171n43

$-152,237 n 326$

$-152-53,158 \mathrm{n} 202$

- 154-55, 161n9

- 156-57, 148n159

$-157,151 \mathrm{n} 170,234 \mathrm{n} 318$

$-158,161 \mathrm{n} 9$

$-159,220 n 258$

$-160,147 \mathrm{n} 154$

- Ms. Munich, 408

- 9b, 120n72

- 10a, 117n57

- 47a, 77n159, 140n133, 185n100

- 87b-88a, 285n134
- Ms. New York, JTS 1813

- 27a-b, $285 n 134$

- 27b, $285 \mathrm{n} 134$

Hayyei ha-'Olam ha-Ba', 86n195, 128, 202-3, 206n199, 212, 220n261, 287, 288, 323, 344-45, 347, 361n13

$-45,50 n 15$

$-48,202 n 180$

$-53,127 n 93$

$-83,333 n 127$

$-122,64 n 87$

- 195-96, 206n199

$-196,212 \mathrm{n} 223$

-196-97, 169n36, 212n224, 228n290

- 197, 264n29

- Ms. Oxford, Bodleian 1582, 51b, 288n152 Imrei Šefer, ed. Gross (Jerusalem: 2001), 83n181, 96, 181, 207n206, 220, 321, 323, 329

$\begin{array}{ll}-8 & 204 n 194\end{array}$

- $16 \quad 147 n 152$

- 18 226n285, 291n172

- 20-21 110n15

$-34 \quad 361 \mathrm{n} 14$

- $37 \quad 207 n 206$

$-40 \quad 207 n 205$

$-50 \quad 87 n 198$

- 60 103n264, 187n106

$\begin{array}{ll}-62 & 225 \mathrm{n} 277\end{array}$

$\begin{array}{ll}-67 & 181 \mathrm{n} 83\end{array}$

$-69333 n 125$

-72 228n292

$\begin{array}{ll}-73 & 101 n 257\end{array}$

$\begin{array}{ll}-86 & 336 n 137\end{array}$

$-10687 n 198$

- 106-7 225n 278

- 121 222n268, 224n276

$-127 \quad 364 \mathrm{n} 25$

- 128-29 96n231

- 129 362n17, 364n25

- 134 130n101

-136 363n20

-150-51 218n246

$-15696$

- 190 187n106, 315n58

- 193-94 94n221, 364n27

- 198 218n246, 220n260

- $202 \quad 202$ n178 
Mafteah ha-Hokhmot, ed. Gross (Jerusalem, 2001), 72n134, 192n129, 257

$-1-2,320 n 78$

$-2,72 n 135$

$-6, \mathrm{~V}, 120 \mathrm{n} 71,209 \mathrm{n} 211,218 \mathrm{n} 251$

$-6-7,115 n 42$

$-7,117 n 54$

$-32,186 \mathrm{n} 105,257 \mathrm{n} 4$

$-38,257 n 7$

$-39,190 n 120$

- 40-41, 316n64

$-41,168 n 33$

$-43,219 n 253$

- 44, 88n203, $115 n 38$

- 44-45, 133n114

$-46,82 n 178$

$-48-49,237 n 325$

$-54,168 n 34$

$-55,168 \mathrm{n} 33$

$-56-57,275 n 79$

$-60,108 \mathrm{n} 4,164 \mathrm{n} 18,180 \mathrm{n} 80,232 \mathrm{n} 307$

$-76,121 \mathrm{n} 75$

$-78,221 \mathrm{n} 265$

$-89,58 n 63$

- 89-93, $123 n 79$

$-91-92,171 n 44$

$-92,172 n 46$

$-101,82 \mathrm{n} 179,120 \mathrm{n} 71$

$-103,220 n 259$

$-105,234 n 318$

- Ms. Parma, de Rossi 141

- 7a, 21-22, 118n60

Mafteah ha-Ra'ayon, 59, 149, 180, 312n41

$-1,136 \mathrm{n} 118$

$-5,184 n 93,196 n 146,234 n 317,360 n 8$

$-23,142 \mathrm{n} 137$

- 23-24, 129n97, 246n360

$-24,148 \mathrm{n} 159,180 \mathrm{n} 81$

$-24-25,59 n 73$

$-25,103 n 263,112 \mathrm{n} 26$

- 43-44, 200n168

$-44-45,265 n 31$

$-45,246 n 360$

$-64,247 n 364,333 n 125$

$-68,119 n 66$

$-69,115 n 38,149 n 161$

$-69-70,14 n 39$

$-70,103$ n269

$-73,333 n 125$

- Ms. Paris, BN 1092, 90a-91b, 59n73
Mafteah ha-Sefirot

$-3,202 \mathrm{n} 178$

$-14,147 n 152$

$-16-17,211 \mathrm{n} 219$

$-20,361 n 13$

$-33,94 n 224$

$-56,218 \mathrm{n} 246$

$-68,154 n 183$

- 68-69, 202n177

Mafteạ̣ ha-Šemot, ed. Gross (Jerusalem, 2001), 84, 134n114

$-16,150$ n168

$-21,213 n 226$

- 25-26, 204n189

- 26, 94n224, 204n190, 262n24

- 28-29, 211n218

$-29-30,168 \mathrm{n} 33$

$-31,190 \mathrm{n} 120$

$-33,205 n 196$

$-39,156 n 195$

$-49,206 n 200$

$-81-82,252 \mathrm{n} 392$

$-86,82 n 177,206 n 201$

$-86-87,108 n 4$

$-87,218 \mathrm{n} 248$

- 100-101, 259n15

$-114,315 n 63$

$-128,316 n 64$

$-147,320 n 78$

$-147-48,275 n 78$

$-148,259 n 14$

$-149,259 n 16$

$-156,16 n 44$

$-163,248 n 374,257 n 6$

- 163-64, 320n79

- 164, 84n184, 157n201

- 164-65, 220n259

- Ms. New York, JTS 843

- 52b-53a, 137n122, 242n350

- 68b, 252n392

Mafteah ha-Tokhahot, ed. Gross (Jerusalem: 2001)

$-23,147 \mathrm{n} 152$

$-62,363 n 20$

$-78,69$

- 122-23, 156n194

-124-25, 220n259

- Ms. Oxford, Bodleian 1605, 46b,78, 216n240 


\begin{tabular}{|c|c|}
\hline Masref la-Kesef, ed. Gross (Jerusalem: 2001), & $1.2 \quad 102$ \\
\hline $117 n 52$ & $-1: 3,188$ \\
\hline$-7-9,261 n 21$ & $-1: 3,192$ \\
\hline$-23,258 n 12$ & $-1: 5,155$ \\
\hline - Ms. Sassoon 56 & $-1: 8,173$ \\
\hline$-24 b, 314 n 50$ & $-1: 8,174$ \\
\hline$-33 b, 98 n 243,186 n 105$ & $-1: 9,176-77 \quad 244 n 357$ \\
\hline$-33 b-34 a, 258 n 12$ & $171 n 44,172 n 45$ \\
\hline Oșar 'Eden Ganuz, ed. Gross (Jerusalem, & $-1: 9,181-82 \quad 179 n 77$ \\
\hline 2002), 70, 72n133, 75, 77-78, 80-81, & $-1: 9,183 \quad 177 n 69$ \\
\hline $96-97,121,146,166,176,182,200,220$ & $-1: 10,185$ \\
\hline $222-23,228,230,262,316 n 64,319,334$, & $-1: 10,185-86 \quad 56 n 52,200 n 167$ \\
\hline $336,363 n 22$ & $-1: 10,185-93$ \\
\hline - preface, $2 \quad 222 n 268$ & $-1: 10,190 \quad 259 n 16$ \\
\hline$-1: 1,5 \quad 243 n 352$ & $177 n 72,223 n 270$ \\
\hline$-1: 1,7-8 \quad 133 n 113$ & $-1: 10,193$ \\
\hline$-1: 1,21 \quad 67 n 96,116 n 47$ & $221 n 264,223 n 270,333 n 125$ \\
\hline$-1: 1,26 \quad 179 n 78$ & $-2: 1,204$ \\
\hline$-1: 1,30-31 \quad 335 n 135$ & 199n162, 211n219, 223n270 \\
\hline$-1: 1,31 \quad 334 n 134$ & $-2: 1,212$ \\
\hline$-1: 1,33 \quad 293 n 176$ & $-2: 1,215$ \\
\hline 291n170 & $-2: 2,224$ \\
\hline $103 n 266$ & 119n66, 130n104, 243n352 \\
\hline $114 n 34,122 n 78,179 n 79$ & $-2: 8,272$ \\
\hline$-1: 2,54$ & $-2: 9,277$ \\
\hline $212 n 222$ & $-2: 9,279$ \\
\hline 150n168 & $57 n 59,97 n 242,284 n 123$ \\
\hline $103 n 271$ & $-2: 9,285 \quad 57 n 59$ \\
\hline $202 \mathrm{n} 180$ & $-2: 9,288-89 \quad 137 n 126$ \\
\hline$-1: 2,90$ & $-2: 9,289 \quad 118 n 59$ \\
\hline 199n163 & $-3: 1,302-3 \quad 96 n 230$ \\
\hline$-1: 2,96$ & $-3: 1,303 \quad 56 n 52$ \\
\hline$-1: 2,103 \quad 199 n 163$ & $-3: 1,305 \quad 96 n 230$ \\
\hline$-1: 3 \quad 151$ & $-3: 1,307-8 \quad 83 n 182$ \\
\hline$-1: 3,110 \quad 80 n 171,122 n 78$ & $-3: 2,311 \quad 83 n 182$ \\
\hline$-1: 3,114$ & $-3: 2,313$ \\
\hline$-1: 3,116$ & $-3: 5,319$ \\
\hline$-1: 3,118$ & $-3: 5,321$ \\
\hline$-1: 3,120$ & $-3: 6,324$ \\
\hline$-1: 3,121 \quad 175 n 61,264 n 28$ & $-3: 7,327$ \\
\hline$-1: 3,121-22 \quad 149 n 165,150 n 168$ & $-3: 7,330$ \\
\hline$-1: 3,122-23 \quad 175 n 61$ & $-3: 7,331$ \\
\hline$-1: 3,123 \quad 264 n 28$ & $-3: 7,332$ \\
\hline$-1: 3,124$ & $56 n 52,227 n 288,259 n 16$ \\
\hline$-1: 3,127$ & $-3: 8,338$ \\
\hline$-1: 3,129$ & $-3: 8,341$ \\
\hline $157 n 197,333 n 125$ & $-3: 9,346-47 \quad 247 n 367$ \\
\hline$-1: 3,133$ & $-3: 9,347 \quad 243 n 352$ \\
\hline$-1: 3,139$ & $-3: 9,349$ \\
\hline$-1: 3,140$ & $-3: 9,352-53 \quad 224 n 274$ \\
\hline
\end{tabular}




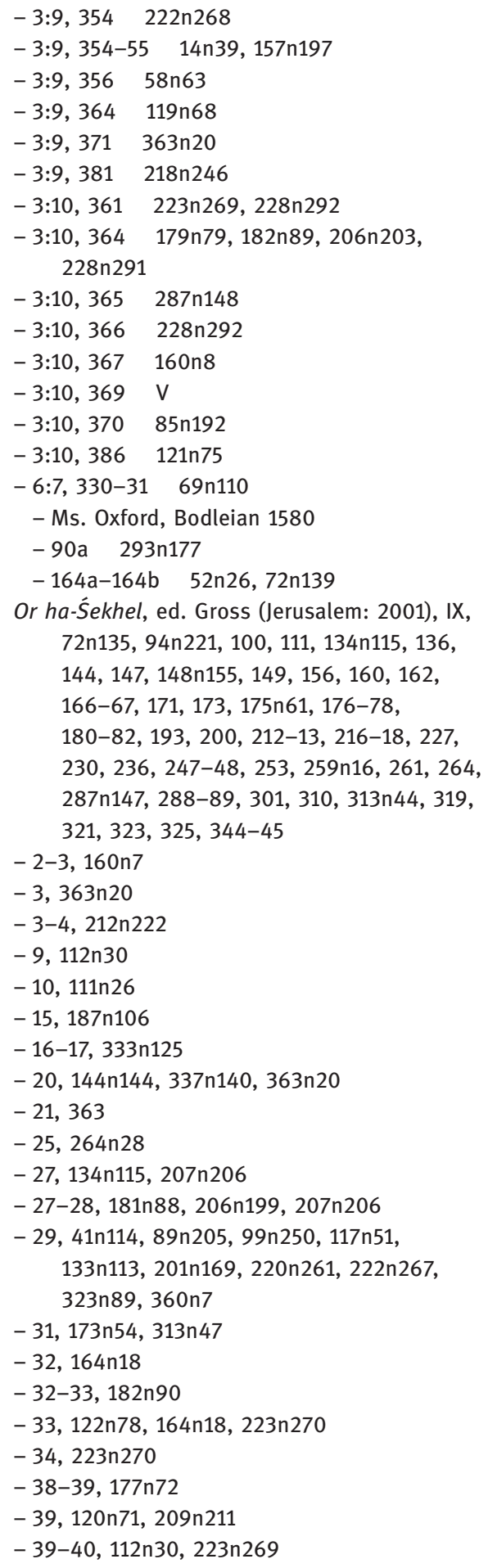

Or ha-Śekhel, ed. Gross (Jerusalem: 2001), IX, 72n135, 94n221, 100, 111, 134n115, 136, 144, 147, 148n155, 149, 156, 160, 162, 166-67, 171, 173, 175n61, 176-78, 180-82, 193, 200, 212-13, 216-18, 227, 230, 236, 247-48, 253, 259n16, 261, 264, 287n147, 288-89, 301, 310, 313n44, 319, $321,323,325,344-45$

$-2-3,160 n 7$

$-3,363 \mathrm{n} 20$

$-3-4,212 n 222$

$-9,112$ n 30

$-10,111 \mathrm{n} 26$

$-15,187 \mathrm{n} 106$

$-16-17,333 n 125$

$-20,144 n 144,337 n 140,363 n 20$

$-21,363$

$-25,264 n 28$

$-27,134 n 115,207 n 206$

- 27-28, 181n88, 206n199, 207n206

- 29, 41n114, 89n205, 99n250, 117n51, 133n113, 201n169, 220n261, 222n267, 323n89, 360n7

$-31,173 n 54,313 n 47$

$-32,164 \mathrm{n} 18$

$-32-33,182 \mathrm{n} 90$

$-33,122 n 78,164 n 18,223 n 270$

$-34,223 n 270$

- 38-39, 177n72

$-39,120 n 71,209$ n211

- 39-40, 112n30, 223n269

- 40, 200n166, 362n15

$-41,165 n 23,267 n 39,359 n 4,364 n 26$

$-45,200 n 166$

$-47,247 n 367$

$-48,247 \mathrm{n} 367$

$-54,180 \mathrm{n} 82$

$-66,207 \mathrm{n} 206$

$-69,112 \mathrm{n} 26$

$-70,247 n 367$

$-70-71,175 n 61$

$-71,187 \mathrm{n} 106$

$-72,193 n 132$

$-72-73,250$ n383

$-76,313 n 49$

$-77,247 n 367$

$-82,165 n 23$

$-84,359 n 6$

$-85,218 n 246,247 n 367$

$-92-93,248 n 369$

$-105,363 n 20$

$-107,175 n 61$

$-108,218 \mathrm{n} 250$

- 108-9, 146n147, 333n125, 362n15

$-110,248 \mathrm{n} 373$

$-111,248 \mathrm{n} 37$

$-119,148 \mathrm{n} 157$

$-120,206 n 199$

- 121, 200n166, $254 n 399$

- ed. Safrin (Jerusalem: 1999), 41-44 166 n27

- Ms. Vatican 233

- 1b-2a, 3 319n76

- 37b-39b 166n27

- 109a 105, 288n153

- Ms. Vatican 597, 113a 288n154

- Ms. Berlin 122, Or. 8 $358,1 a-59 b$ 325n98

Peruš Sefer ha-Hafțarah, 158, 249-50

- in Mașref ha-Śekhel

- 107, 250n385

$-111,352 \mathrm{n} 206$

$-113,249$ n383

- 117, 186n104, 246n364

- Ms. Roma, Angelica 38, 108n6

- 9a, 119n66, 209n211, 214n229

- 37a, 249n383

Sefer Gan Na'ul

- 2, 146n151

$-5,16 n 44$

- 40-41, 211n219 
$-58-59,148 \mathrm{n} 158$

Sefer Ge'ulah, 60n75, 119n66

$-5,112 \mathrm{n} 27$

$-5-6,109 n 14$

$-11,193 n 131$

$-14,313 n 46$

$-14-15,110$

$-32,185 n 102,319 n 77$

$-36-37,58 n 62$

$-37,96 n 236$

$-38,23 n 57$

$-45,237 n 325$

- Ms. Jerusalem, NUL, $8^{0}$ 1303, 73a, 109n14

Sefer ha-Hešeq, ed. Gross (Jerusalem: 2002), 72n136, 191-92, 220, 288n155, 319, 323

- 1, 218n248, 220n259, 222n267

$-2,72 \mathrm{n} 136$

$-3,333 n 125$

$-10,333$

$-16,260 n 18,287 n 148$

$-28,196 n 146$

$-33,333 n 125$

$-34,187 \mathrm{n} 106$

$-39,86$ n195

$-42,228 \mathrm{n} 293,333 \mathrm{n} 125$

$-54,332 n 120,334 n 134$

$-61,220 \mathrm{n} 257$

$-70,315 n 63$

- 80, 220n257, 334n131, 361n12 - ed. Safrin (Jerusalem: 1999), 7 84n185

- Ms. New York, JTS 1801

- 8b, 191n123

- 19b-20a, 191n123

Sefer ha-Melammed, ed. Gross (Jerusalem, 2002), 171n41, 187, 192, 201

$-6,103$

$-6-7,248 \mathrm{n} 368$

$-9,364 n 25$

$-16,221 \mathrm{n} 265$

$-19-20,284 \mathrm{n} 121$

$-23,57 n 59$

$-24,170 n 41,175 n 62$

$-24-25,188 \mathrm{n} 117$

$-26,192 \mathrm{n} 130$

Sefer ha-Ot, ed. Jellinek (Breslau, 1887), 13, 167,349

$-65,329 n 112$

$-69,259 n 13$

$-76,138 \mathrm{n} 129$

$-79,108 n 7$
$-82-83,313 n 49$

$-84,168 \mathrm{n} 31$

$-85,168 \mathrm{n} 32$

Ševa' Netivot ha-Torah, ed. Jellinek (Leipzig: Heinrich Hunger, 1854), 52, 62, 95, 105, 146n147, 322

$-1: 14 \quad 52 \mathrm{n} 27$

$\begin{array}{ll}-1 & 74 \mathrm{n} 147\end{array}$

- 4 103, 104n272, 179n77, 222n268, 235n321, 319n75

$\begin{array}{ll}-5 & 322 n 85\end{array}$

$\begin{array}{ll}-7 & 322 n 85\end{array}$

$\begin{array}{ll}-8 & 179 n 77\end{array}$

- 9 204n192, 222n268, 223n270

- $11322 \mathrm{n} 85$

$-12 \quad 218 n 246$

$-13 \quad 333 n 125$

- $1456 n 56,96,175 n 60$

-14-15 82n180, 96n236

$-16-17 \quad 313 n 45$

$-19333 n 125$

- $21 \quad 67 n 98,68 n 100,103 n 264$

$-2255 n 48,70 n 111$

$-23 \quad 349 n 189$

- $25196 \mathrm{n} 146$

Sitrei Torah, 25, 60n75, 70, 73, 94, 136n117, 153, 165n23, 167n30, 176, 202-3, 209, 212, 218n247, 220n261, 222n267, 232, 243n352, 301, 319, 344-45

$-7,83 n 181$

$-11,69$ n109

- 11-12, $234 \mathrm{n} 318$

$-14,85 n 190,113 n 32$

$-16-17,265 n 30$

$-17,352$ n209

$-17-19,185 n 102$

$-20,99$ n 250

- 22-26, 210n215

$-32,56 n 51$

$-33,82 \mathrm{n} 179$

$-34,191 \mathrm{n} 123$

$-35,112 \mathrm{n} 26$

- 35-36, 56n56, 203n185

$-36,202 \mathrm{n} 181$

$-36-37,178 n 74$

$-38,212$ n 220

- 44, 297n185

$-61,69 n 109,221 \mathrm{n} 265$

$-69,284 \mathrm{n} 124$

$-70,116 n 44,154 n 186$ 
- 71, 194n140, 195n141

$-73,96 n 229,259 n 16$

$-75,359 \mathrm{n} 4$

$-78,86 n 195$

$-90,257 \mathrm{n} 1$

$-91,275 n 78$

- 91-92, 111n20

$-92,172 \mathrm{n} 50$

- 111, 198n154, 222n267, 360n8

$-113,363 n 20$

$-115-16,364 n 25$

$-118,112$ n 30

$-122,361 \mathrm{n} 13$

$-125,103 n 263$

$-137,363 n 20$

- 140, 86n195, 244n358

$-141,153 n 181$

$-146,176 n 64$

$-152,147 \mathrm{n} 154$

$-154,86 n 195$

- 160, 96n233, 203n187, 293n177

$-161,314 n 56$

$-167,151 \mathrm{n} 170$

$-168-69,61 \mathrm{n} 76$

$-170,363 n 20$

$-174,316 n 64$

$-175-76,29 n 78$

$-186,113 n 31$

- 188, 228n289, $264 \mathrm{n} 29$

$-194,86 \mathrm{n} 195$

- 195-96, 136n120

$-199,75 n 152$

- Ms. Paris, BN 774

- 120a, 17 51n22

- 120a, 17-18 74n152

-125 a, 139 181n87

$-148 \mathrm{~b}, 116 \quad 362 \mathrm{n} 17$

- 155a, $138 \quad 227 n 288$

- 162a, 158 176n65

- Ms. Munich, 341, 160b 210n215

Sod Yerušalayyim, ed. Raphael Kohen (Jerusalem: Raphael Kohen, 2001)

- 16, 243n355

Šomer Mișwah, 50n17

$-5-6,232 n 308$

$-11,68 \mathrm{n} 104$

$-12,137 n 123$

-14-15, 259n16, 262n24

$-15,221 n 264$

$-16,244 n 356$
$-27,58 n 68,69 n 109$

$-28,339 n 151$

$-40,252 n 389$

$-41, \mathrm{~V}$

$-42,119 n 64,146 n 148$

$-47,315 n 63$

$-118,146$ n152

- Ms. Paris, BN 853, 57a,24, 220n258

Untitled Treatise (Ms. Firenze, Laurenziana, Plut. II. 48), 152, 156, 178, 214-15, 261, 349n189

$-69 a, 156,278 n 90$

- 70a, 246n364

- 70b, 193n132, 194n140, 333n126

- 71a, 156n193, 262n25

$-71 \mathrm{~b}, 156 \mathrm{n} 192,278 \mathrm{n} 90$

$-74 \mathrm{~b}, 152 \mathrm{n} 174$

$-75 a, 333 n 126$

$-75 b, 193 n 132,333$

$-76 a, 156 n 191$

$-77 a, 153 n 179$

$-78 \mathrm{a}, 193 \mathrm{n} 132$

$-78 b, 314 n 55$

$-79 a, 156 n 191$

- 79b, 127n93, 333n126

- 80a, 227n288

$-81 a, 252 n 394$

- 82b, 195n143

- 83a, 228n289

- 83b, 196n146

- 87b-88a, 263n27

- 89a, 155n190, 195n143

- 89b-90a, 156n192, 278n90

- 90b, 155n190, 333n126

- 91a, 195n142

- 92a-b, $152 \mathrm{n} 179$

- 92b, 152n175

- 93b, 152n175

- 94b, 170n40

- 95a, 156n191, 214n233

$-95 \mathrm{~b}, 314 \mathrm{n} 55$

- 97a, 152n175

- 98b, 261n22

- 99a, 313

- 99b, $178 \mathrm{n} 74$

We-Zot li-Yehudah, ed. Adolph Jellinek (Leipzig: A.M. Colditz, 1853), 57, 66, 71n121, 103, 329, 332

$-16 \quad 181 n 84$ 


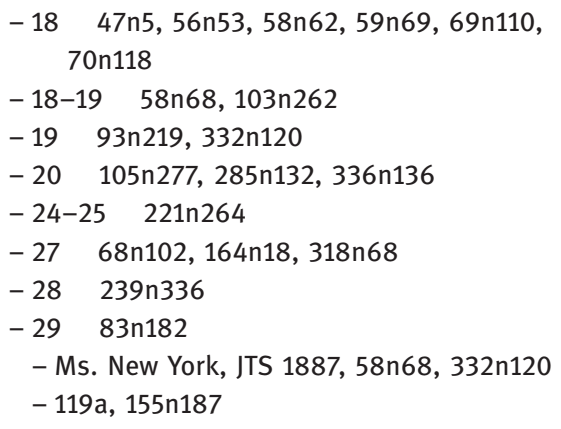

\section{Abraham Abulafia's school (anonymous)}

Commentary on the Pentateuch, Ms. Oxford, Bodleian 1920

- 16a, 340n152

Ner Elohim, ed. Gross (Jerusalem: 2002), 40n112, 227n288, 261n21, 362

- $29 \quad 333 n 125$

- 46 190n122, 194n140

$-48 \quad 118 n 58$

$-72 \quad 99 n 250$
-96

- $96 \quad 122 \mathrm{n} 77$

- Ms. Munich 10

- 154b, 363n21

- 156b-157a, 265n31

Sefer ha-Șeruf, ed. Gross (Jerusalem, 2004)

$-1,257 n 3$

$-11,243 n 355$

- 24-25, 157n199

- 153, 318n69

Sefer Or ha-Menorah, Ms. Jerusalem, $8^{0} 1303$

- 26b-27a, 156n192

- 28b, 202n180, 23z1n306

$-43 a, 202 n 180$

\section{Abraham Ibn Ezra}

Sefer ha-Šem, 103-4

- Chapter 3, 247n364

Sefer ha-Ṣaḥut, 103, 247n364

\section{Anonymous}

Sefer Razi'el, 68

Sefer Toledot Adam

- Ms. Oxford, Bodleian 836

- 154a, 261n21

- 156a, 262n26

$-157 b, 115 n 41$

- 159a, 148n157
- 165a, 220n261, 227n288

-165b, 200n166

- 166ab, 198n156

- 167b, 200n166

$-169 a, 115 n 40$

- 171b-172a, 147n154

The Book Bahir, 8, 68, 99, 321, 335n135

\section{Anonymous (follower of Solomon ibn Adret)}

Ma'arekhet ha-Elohut (Mantua: 1558), 340

- 86b, 340n154

\section{Azriel of Gerone}

Commentary on the Talmudic Legends, ed. Isaiah Tishby (Jerusalem: Nekize Nirdamim, 1945)

-14, 267n39

\section{Bahyya ben Asher}

Bahya on the Torah, ed. Chaim D. Chavel (Jerusalem: Mossad ha-Rav Kook, 1967)

$-2,341 n 158$

$-104-5,283 n 116$

- 268, 283n116

- 352, 341n157

Kad ha-Qemah, Lulav, in Kitvei Rabbenu Bahya, ed. Chaim D. Chavel (Jerusalem: Mossad ha-Rav Kook, 1970), 234, 282

\section{Bahya ibn Paqudah}

Hovot ha-Levavot (trans. Judah ibn Tibbon), $58 \mathrm{n} 62$

- Gate 10, chapter 1, 558, 323n89

\section{Baruch Togarmi}

Commentary on Sefer Yeșirah, printed in Scholem, The Kabbalah of Sefer ha-Temunah, $52,68,143,184,194,220 \mathrm{n} 257$

$-234,247 n 364$

$-236,313 n 46$

$-238,313 n 46$

\section{Bedershi, Abraham ben Isaac}

Hotam Tokhnit, ed. Gavriel Pollak (Amsterdam: Levison and Proops, 1865)

$-1.24,265,118 n 63$ 


\section{Elijah of London (attributed to)}

Ms. Sassoon 290

- $381290 n 162$

$-648 \quad 290 n 168$

\section{Hananel ben Abraham Esquira}

Sefer Yesod 'Olam, Ms. Moscow, Ginsburg 607

- 77a, 247n364

- 79b, 195n140

\section{Ḥayyim Vițal}

Ša'arei Qedušah (Jerusalem: 1973)

$-7,288 n 156$

(attributed to)"The Fourth Part of Sha'arei Qedushah," in Ketavim Hadašim me-Rabbenu Hayyim Vițal, ed. Nathanel Safrin (Jerusalem: Ahavat Shalom, 1988)

$-10,290 n 165$

\section{Hillel of Verona}

First letter to Maestro Gaio, 54n42, 61-62

Printed in Ta'am Zeqenim, ed. Eliezer Ashkenazi (Frankfurt am Main: 1854)

- 70b-73a, 53n34

$-72 \mathrm{a}, 61 \mathrm{n} 78$

Tagmulei ha-Nefeš (ed. Joseph B. Sermoneta), 55, 61n77, 62, 65, 97

$-56,119 n 66,213 n 226$

$-72,213 n 226$

$-73,97 \mathrm{n} 238,213 \mathrm{n} 226$

$-73-100,146 \mathrm{n} 152$

\section{Isaac Albalag}

Sefer Tiqqun ha-De'ot, ed. Georges Vajda (Jerusalem: Israel Academy of Sciences and Humanities, 1973)

$-58,156 n 191$

$-60,156 \mathrm{n} 191$

$-77-78,183 n 93$

$-82-83,31 n 90$

$-83-84,138 n 126$

\section{Isaac Ben Samuel of Acre}

Sefer Oșar Hayyim

- Ms. Moscow, Ginsburg 775

- 93a, 233n316

$-233 b, 233 n 315$
- Ms. Oxford, Bodleian 1911

-154b, 233n315

Jacob Ben Sheshet

Mešiv Devarim Nekhohim, 26n69

\section{Jacob Joseph of Polonnoye}

Sefer Șafnat Pa'aneah, ed. Gedalya Nigal (Jerusalem: ha-Makhon le-Heqer ha-Sifrut haHasidit, 1989)

$-260,88 n 204$

\section{Joseph ben Shalom Ashkenazi}

Commentary on Genesis Rabbah, ed. Moshe Hallamish (Jerusalem: Magnes Press, 1984)

$-40,43 n 118$

$-80,185 \mathrm{n} 100$

$-102,208 \mathrm{n} 208$

$-142,188 \mathrm{n} 112$

$-146,28 \mathrm{n} 75$

$-146-47,56 \mathrm{n} 52$

$-250,28 n 75$

$-269,27 n 73$

$-274,43 n 118$

Commentary on Sefer Yeșirah (Jerusalem, 1961)

$-6 a, 27 n 73$

$-9 \mathrm{c}-\mathrm{d}, 27 \mathrm{n} 73$

- 31d, 87n201

$-44 d, 29 n 80$

$-55 c, 88 n 201$

\section{Joseph Gikatilla}

Commentary on Ma'aśeh Berešit

- Ms. New York, JTS 1891

- 70a-b, $194 n 140$

- Ms. New York, JTS 2156

- 39a, 190n122

Ginnat Egoz (Jerusalem: Yešivat Ahavah weḤayyim, 1989) 76, 106, 247n364, 269n45, $325 \mathrm{n} 97$

$-34,190 \mathrm{n} 122$

$-98,154 n 185$

$-168,42 \mathrm{n} 117$

- 340, 247n364

- 337, 194n135

- 340-41, 42n117

- 343-45, 247n364 
- 345-47, 42n117

Ginnat Egoz (Hanau, 1615)

- 5c-d, 193n132

- 12d, 193n132

- 13a, 193n132

- 13b, 193n132

\section{Joseph ibn Kaspi}

Adnei Kesef, ed. Isaac H. Last (London: Narodiczky, 1912)

$-2: 75,87 n 200$

- 2:140, 84n186

Commentary on Proverbs, printed in 'Aśarah Kelei Kesef

- 1:17, 130n100

- 1:19, 185n100

Maśkiyyot ha-Kesef

ed. S. Werbluner (Frankfurt am Main, 1848), reprinted in Šelošah Qadmonei Mefaršei haMoreh (Jerusalem: 1961)

$-74-75,20 \mathrm{n} 50$

- 109-10, 23n57

Menorat Kesef, printed in 'Aśarah Kelei Kesef, ed. Isaac H. Last (Pressburg: Alcalay, 1903)

$-81,94 \mathrm{n} 224$

$-94,87 n 200$

- 2:100-101, 84n186

$-2: 103,84 n 186$

- 2:108, 84n186

Sefer ha-Mussar, printed in 'Aśarah Kelei Kesef - 2:60, 87n200

Šulhan Kesef, ed. Hannah Kasher (Jerusalem: Ben Zvi Institute, 1996), 37

\section{Judah Alboțini}

Sullam ha-'Aliyyah, ed. Joseph E. E. Porush (Jerusalem: 1989)

$-56,190 n 122$

$-69,289 n 158$

- 71, 287n142

- 73, 229n296, 288n152

\section{Judah ben Nissim ibn Malka}

Kitāb Uns wa-Tafsīr, ed. Yehuda A. Vajda (Ramat-Gan: Bar-Ilan University Press, 1974)

$-53,288 n 151$

\section{Judah Ha-Levi}

Kuzari, 67, 104n274, 141, 261n20, 300, 302

$-1: 103,267 n 39$

$-4: 3,247 n 364$

$-4: 25,67$

Judah Ḥallewah

Sefer Șafnat Pa'aneạ̣

- Ms. Dublin, Trinity College, B. 5. 27

- 15a, 312n41

\section{Judah ibn Matkah}

Midrash Hokhmah, 39, 97

\section{Judah Romano}

Commentary on the Account of Creation Ms. Firenze, Laurenziana, Plut. I, 22

- 45a, 28n77

\section{Levi ben Abraham}

Battei ha-Nefeš we-ha-Lehašim, 39

Liwyat Hen, Ma'aśeh Berešit (Jerusalem: The World Union of Jewish Studies, 2004), $37 n 99$

$-38-39,160 n 5$

$-135-36,39 n 108$

- 367-68, 39n108

Liwyat Hen: The Quality of Prophecy and the Secrets of the Torah

- 212, 113n32

Liwyat Hen: The Work of the Chariot

-95-96, 40n112

$-133,39 n 108$

-187-88, 283n118

- 250-63, 187n107

$-287,283 n 118$

\section{Menahem Recanati}

Commentary on the Torah (Jerusalem: 1961), 340

$-25 d, 340 n 153$

- 66a, 340n153

\section{Moses Cordovero}

Or Yaqar, vol. 5 (Jerusalem: Ahuzat Yiśra'el, 1970)

$-18,340$ n152 
- 194, 340n152

- 245, 340n152

Or Yaqar, vol. 11 (Jerusalem: Aḥuzat Yiśra'el, 1981)

$-113,340 n 152$

\section{Moses de Leon}

The Book of the Pomegranate, ed. Elliot R. Wolfson (Atlanta: Scholars Press, 1988)

- 36-38, 42n116

- 390-92, 42n116

\section{Moses ibn Tibbon}

Sefer Pe'ah, eds. Howard Kreisel, Colette Sirat, and Avraham Israel (Beer-Sheva: Ben-Gurion University Press, 2010)

- 99, 84n186, 156n191, 169n38

$-102,156 n 191$

$-103,323 n 89$

\section{Moses Maimonides}

The Guide of the Perplexed, XIII-XIV, 12, 16-18, 20n50, 21-23, 25-26, 32-40, 46, 49, 51-67, 69-71, 73-77, 78n165, 79, 83-90, 92-94, 99-101, 103, 112n30, 120, 128, 132-33, 137, 142, 159, 162-63, 176, 185-86, 200n169, 204-5, 209-10, 212n225, 213n228, 215, 226n285, 227, 255, 257n2, 265, 296, 300-1, 318, 326-27, 345, 348, 359

- 1:2, 128n94

$-1: 9,193 n 132$

$-1: 10,202 n 179$

$-1: 11,138$ n126

- 1:14, 119n67, 222n268, 232n310

$-1: 46,187 n 110,188 n 116$

$-1: 60,254 n 400$

$-1: 61,359 n 3$

$-1: 65,177 n 71$

- 1:66, 187n110, 188n116, 198n156

$-1: 70,201 n 171,313 n 46$

$-1: 72,20 n 50,190 n 120$

- 1:73, 150n166, 219n254, 221n265

$-1: 74,173$

$-2: 10$, XIV

- 2:12, XIIIn11

- 2:30, XIIIn11, XVn22, 126n89, 128, 132n111, $188 n 112$

$-2: 32,207 n 205$
$-2: 43,23 n 57$

$-2: 45,144 \mathrm{n} 142$

$-2: 48,164 \mathrm{n} 20$

- 3:22, XIIIn11

- 3:27, 117n51

- 3:51, 75

- 3:54, 109n13

Mishneh Torah, 69, 170n40, 172n46, 333n126, $363 n 20$

Hilekhot Melakhim

- 4:10, 170n40

$-10: 9,170 n 40$

- 12:10, 17040 Hilekhot Yesodei ha-Torah

- 2:10, 333n126

$-4: 6,228 n 289$

$-7: 1,228 n 289,363 n 20$

$-9: 2,170 n 40$

- 71:1, 170n40

\section{Moses Narboni}

Commentary on the Guide, ed. Jakob Goldenthal (Vienna: K.K. Hof- und Staatsdruckerei, 1852)

$-15 b, 38 n 105$

- 16b, 20n50

\section{Moses of Kiev}

Šušan Sodot (Koretz: 1784)

- 69b, 320n82

\section{Nahmanides}

Kitvei ha-Ramban, ed. Chavel (Jerusalem, Mosad ha-Rav Kook 1963)

- 1:154, 207n207

$-1: 158,207 n 207$

- 1:291, 351n201

$-1: 294,351 n 201$

- 1:306, 349n191

Commentary on the Pentateuch (ed. Chavel), 207n207, 341

- 104-5, 283n116

\section{Nathan ben Sa'adyah Ḥar'ar}

Ša'arei Ședeq, In Le Porte della Giustizia, trans. Maurizio Mottolese, ed. Moshe Idel (Milan: Adelphi, 2001), 201n170, 205, 
232-33, 286-88, 313n46, 320, 322, 324, 338

$-55-56,242$ n348

$-373,233 n 314$

- 385, 232n310, 233n312

- 462, 144n142, 338n143

- 462-63, 286n138

$-463,206 \mathrm{n} 204$

- 464-65, 286n139, 287n141

$-465,52 \mathrm{n} 24,286 \mathrm{n} 137$

$-467,287 \mathrm{n} 141$

- 468-69, 287n142

- 469, 205n196, 313n46

$-470,96 n 232$

$-471,205 n 196$

$-473,205 n 196$

$-474,313 n 46$

- 475, 205n196, 287n141

- 476, 152n172, 286n137, 287n146, 361n12

- 477, 111n25, 205n198, 289n157

- 477-78, 52n24, 205n196

- 478, 28n76, 49n10, 205n197, 322n87, 349n189

- 478-79, 241n346, 268n42, 278n92, 322n88

- 479, 205n196, 287n149

$-480,205 n 196$

- 481, 205n196

\section{Nissim ben Moses of Marseilles}

Ma'aśeh Nissim, ed. Howard Kreisel (Jerusalem: Mekize Nirdamim, 2000), 37n99

$-438,39 n 108$

\section{Shem Tov ibn Falaquera}

De'ot ha-Filosofim, 39

Moreh ha-Moreh ed. Yair Shiffman (Jerusalem: World Union of Jewish Studies, 2001), 37, 90

- 186, 20n50

Sefer ha-Ma'alot ed. Ludwig Venetianer (Berlin: S. Calvary, 1894)

$-22,229 n 295$

$-28,119 n 66$

$-50,363 n 20$

- 77, 237n325

\section{Solomon Ibn Adret}

responsum 1 , no. 548, 28n76, 59n69, 184, 205n197
Yohanan Alemanno

Collectanea (Ms. Oxford, Bodleian 2234)

- 95a-96b, 206n199

- 148a-149a, 206n199

Hešeq Shlomo (Ms. Oxford, Bodleian 1535)

- 147a, 206n199

\section{Zeraḥyah Ḥen}

Oșar Neḥmad, ed. Isaac Blumberg (Vienna: Israel Knopfmacher und Sohne, 1857)

- 2:121-22, 71n121

$-2: 125,62 n 81$

$-2: 126,63 n 82$

\section{Christian Sources}

New Testament

1 Corinthians

- 15:45-49, 155n188

\section{Greek Authors}

Aristotle

Metaphysics, 56n52

Meteorology, 57

On the Soul, 57

- 430a16, 160n6

Organon, 56n52, 96

- Categories, 96

- De Interpretatione, 95

- 16a, 163

Physics, $61 \mathrm{n} 77$

Politics, 232

\section{Plotinus}

Enneads, 229

- 4.8.1, 229n294

\section{Proclus}

Elements of Theology, 94

\section{Arabic Authors}

Liber de causis, 94, 364

Theology of Aristotle, 229

- chapter 8, 20n50 


\section{Al-Bațalyawsī}

Sefer ha-'Aggulot ha-Ra'ayoniyyot (trans.

Moses ibn Tibbon), ed. D. Kaufmann (Budapest: S.n., 1880)

$-50,362 n 15$

\section{Al-Fārābī}

Al-Siyāsah al-Madaniyyah, 94

\section{Averroes}

Incoherence of Incoherence

Eng. trans. by Simon Van den Bergh, 2 vols, E.J.W. Gibb Memorial Series (London: Oxford University Press, 1954)

$-1: 224-66,184 \mathrm{n} 93$

$-1: 253-54,20 n 50$

$-1: 272,18 n 47$

$-2: 95,18 n 47$
$-2: 143,20 n 50$

\section{Latin Authors}

\section{Dante Alighieri}

De vulgari eloquentia (Dante Alighieri, De l'èloquence en vulgaire, trans. Irène RosierCatach [Paris: Fayard, 2011])

- 44-45, 171n42

$-115,171 n 42$

\section{Roger Bacon}

"Opus Tertium," in Opera Inedita, ed. John S. Brewer, vol. 1 (London: Longman Green, 1859)

$-47,350 n 198$

$-86,350 n 197$ 